\title{
Toward an insect-inspired event-based autopilot combining both visual and control events
}

\author{
T. Raharijaona ${ }^{1}$, J. Serres ${ }^{1}$, E. Vanhoutte ${ }^{1}$ and F. Ruffier ${ }^{1}$
}

\begin{abstract}
This paper presents the autopilot and the behavior of a "simulated bee" traveling along two different tunnels using both visual and control events. The computational gain of an event-based PID controller compared to its time-based version is usually discussed because the event detector is computationally expensive. By combining visual and control events, the newly suggested event-based autopilot requires very low computational resources. In particular, the event detector which computes the control error and tests its magnitude is activated only when a new contrast is detected by the optic motion detectors that assesses the optic flow, i.e. only when the magnitude of the optic flow error could have changed. This new event-based control strategy used faithfully the visual information already available in the optic flow sensor to reduce even further the computational cost. The "simulated bee" was equipped with: (i) a minimalistic compound eye comprising 10 or 8 local motion sensors (depending of the tunnel configuration) measuring the optic flow magnitude, (ii) two optic flow regulators updating the control signals whenever specific optic flow criteria changed and (iii) three event-based controllers taking into account both error signals and visual events, each one in charge of its own translational dynamics. The "simulated bee" managed to travel safely along the tunnels without requiring any speed or distance measurements, using very low computational resources, by (i) concomitantly adjusting the side thrust, vertical lift and forward thrust only when both a visual contrast and a change of optic flow control error were detected, and (ii) avoiding collisions with the surface of the tunnels and decreasing or increasing its speed, depending on the clutter rate perceived by motion sensors.
\end{abstract}

\section{INTRODUCTION}

The performance of an event-based controller in terms of computational load is usually controversial since the eventdetector criterion is periodically calculated. In a classical event-based PID controller, the event detector requires many computations such as to calculate error and to compare its magnitude at each time step. The idea is to take benefit of event-based nature of the bio-inspired optic flow sensor to perform the autopilot computation only when it is needed. Insect-size Micro Aerial Vehicles (MAV) are increasingly becoming a reality ([1], [2], [3], [4]) and have to be fitted with sensors and flight control systems enabling them to perform any kind of aerial maneuvers extremely close to obstacles both indoor and outdoor. Consequently, moving jointly very close to obstacles and at a high flight speed represents a big challenge for MAV applications. For the purpose of MAV

This work was supported partly by CNRS (Life Science; Information Science; Engineering Science and Technology), Aix-Marseille University and the French Defence Agency (DGA).

${ }^{1}$ T. Raharijaona, J. Serres, E. Vanhoutte and F. Ruffier are with Aix Marseille Univ, CNRS, ISM, Marseille, France thibaut.raharijaona@univ-amu.fr applications with scarce resources, computationally-frugal methods would improve the biomimicry of the optic flowbased autopilot. Winged insects do not require any sensory system such as a GPS or an Inertial Measurement Units (IMU), nor any emissive proximity sensors such as ultrasonic or laser range finders. On the other hand, they rely heavily on Optic Flow (OF), which is the angular velocity of contrasted points, edges, or surfaces caused by the relative motion between the observer and the surrounding objects ([5], [6]). They make use of the OF to jointly avoid walls and adjust their speed in tunnel-like environments with either variable section ([7], [8]), an abrupt change in tunnel width ([9]), a sudden change in unilateral optic flow by manipulating the wall texture from a checkerboard to axial stripes [10]. We can therefore take inspiration from their OF-based strategies to develop smart MAVs autopilots ([11], [12], [8], [13], [14]). Electrophysiological studies on flies motion sensitive neurons have shown that their OF sensitivity is texture- and contrastindependent as well as it can be explained by a "time-oftravel" scheme ([15]).
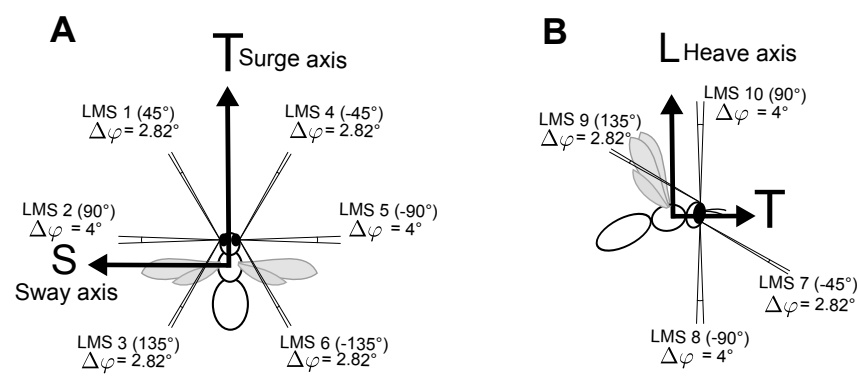

Fig. 1. (A) Top view describing the orientation of 6 Local Motion Sensors (LMSs) in the horizontal plane corresponding to 12 simulated photoreceptors. (B) Side view showing the orientation of 4 LMSs in the vertical plane corresponding to 8 simulated photoreceptors.

The output of "time-of-travel" scheme can be only updated when a change in the optical contrast with time is detected ([15], [16]). An asynchronous signal is therefore generated in the output of a "time-of-travel" algorithm, which can be conveniently used in event-based control systems ([17]). Because of the slight delay in the camera read-out, event-based collision avoidance algorithms have been only developed so far under open loop conditions in slightly cluttered corridors, but they were expected to be ready for use in the near future in closed loop on-board robotic platforms ([18], [19]). Event-based control systems have also been used for visual odometric purposes in the field of robotics ([20]). 


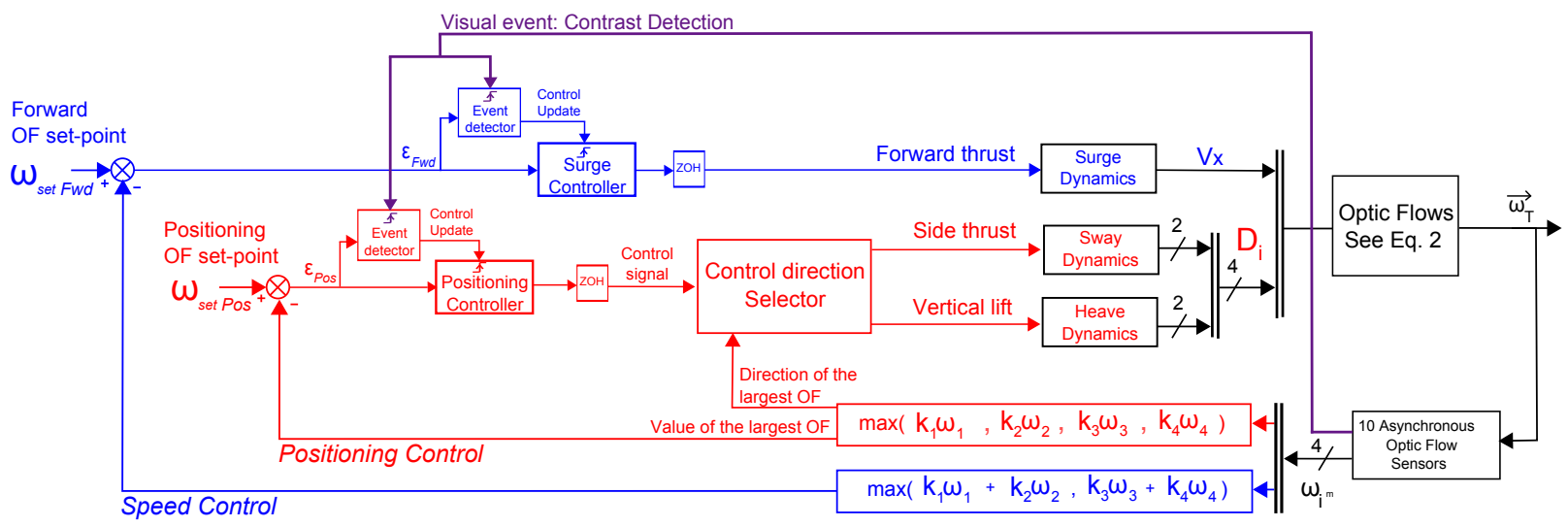

Fig. 2. A visual event which consists of a contrast detection activates the event detector of each visual feedback loop. A visual feedback loop owns its OF set-point: a speed control loop (in blue) and a positioning control loop (in red). The event-based surge controller adjusts the pitch angle $\theta_{\text {pitch }}$ as presented in Fig. 1A (that determines $V_{x}$ via the bees' surge dynamics) on the basis of whichever sum of the two coplanar (horizontal or vertical) OFs

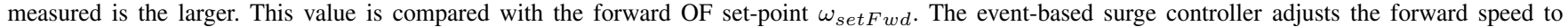
minimize the error $\epsilon_{F w d}$. The event-based positioning controller adjusts the roll angle $\theta_{\text {roll }}$ (or the wing stroke amplitude $\Delta \Phi$ ), which determines the distances to the walls, the ground, or the roof, depending on the sway (or heave) dynamics on the basis of whichever of the measured OFs is the largest. The latter value is compared with the positioning OF set-point $\omega_{\text {setPos }}$. The direction of avoidance is given by a Control direction Selector that multiplies the control signal by a direction factor depending on the direction of the maximum OF signal (see in Fig. 3).

In the present paper, we describe in detail an eventbased version of the ALIS (ALIS stands for "AutopiLot using an Insect-based vision System") which was originally designed with fixed-step digital controllers to perform tunnelfollowing tasks ([21]). Preliminary results were presented in a work-in-progress paper ([22]), however, in this new version, the event-based ALIS autopilot is described in detail including new simulation results showing its ability to fit with bees' behavior and bees' compound eye by combining both visual and control events.

In section 2, the simulation set-up and the simulated agent mimicking a honeybee fitted with a minimalistic visual system including only 10 local OF measurements are described. In section 3, the ALIS autopilot and the event-based discrete time controllers are described in detail. In section 4 , we describe the trajectories of the simulated agent fitted with the event-based ALIS autopilot in a doubly tapering tunnel (with a tapering angle of $7^{\circ}$ in both planes) or in straight tunnel with an abrupt change in width. The results of the simulations performed show that the event-based ALIS autopilot generates a small number of events making the "simulated bee" travel along tunnels endowed with crosssection changes, thus greatly decreasing the number of control signal computations required while mimicking bees' behavior during tunnel-following task.

\section{SIMULATION SET-UP}

\section{A. Software set-up}

In-silico experiments were carried out on two standard computers running: (i) a first one equipped with the MATLAB/Simulink ${ }^{\circledR}$ software program at a sampling rate of $1 \mathrm{kHz}$ for $\mathrm{OF}$ sensing purposes and feeding the eventbased control systems, and (ii) a second one equipped with the MORSE software program ${ }^{1}$ (MORSE stands for "Modular OpenRobots Simulation Engine") to simulate the visual interactions with the virtual 3D environment. The two computers were linked together by a TCP/IP protocol in which the maximum delay was limited to $3 \mathrm{~ms}$. The virtual 3D environment was built with the Blender software program $^{2}$. The four surfaces of the tunnel were lined with high resolution photographs of natural panoramic scenes ([23]). The 20 scalar photoreceptor signals were transmitted via TCP/IP to a second computer processing the 10 local OF computations (Fig. 1B-C), the 3 event-based controllers and the 3 translational dynamics of the "simulated bee" using the Matlab/Simulink software program (Fig. 1A). The Matlab/Simulink program then sent the 3D position $(x, y, z)$ of the "simulated bee" back to the MORSE simulator.

\section{B. Optic flow}

The OF vector field perceived by a simulated agent depends in particular on the structure of the 3D environment ([5], [6]). The OF can be defined by a vector field of the apparent motion of objects, surfaces, and edges in a visual scene generated by the relative motion between an agent and the scene. The OF field $\vec{\omega}(1)$ is a combination between two components: a translational $\overrightarrow{\omega_{T}}$ and a rotational OF $\overrightarrow{\omega_{R}}([6])$.

$$
\vec{\omega}=\overrightarrow{\omega_{T}}+\overrightarrow{\omega_{R}}
$$

It has been established that hymenopterans stabilize their gaze by compensating for any body rotations ([24]). It is assumed that the head of the artificial flying insect is perfectly stabilized canceling all rotation due to body pitch and roll with respect the inertial frame and hence stabilized

\footnotetext{
${ }^{1} 19$ May 2015: MORSE release 1.3 under GNU GPLv2, https://www.openrobots.org/wiki/morse/

${ }^{2} 15$ Nov. 2015: Blender release 2.76 under GNU GPLv2, https://www.blender.org/
} 
along the tunnel axis. Therefore, the head of the artificial flying insect experienced only translational $\mathrm{OF}$ and each $\mathrm{OF}$ sensor will receive a purely translational OF $\left(\overrightarrow{\omega_{R}}=\overrightarrow{0}\right)$. The translational OF (expressed in $\mathrm{rad} / \mathrm{s}$ ) can be defined as follows:

$$
\overrightarrow{\omega_{T}}=-\frac{\vec{V}-(\vec{V} \cdot \vec{d}) \cdot \vec{d}}{D}
$$

where $\vec{d}$ is a unit vector describing the viewing direction, $\vec{V}$ is the translational velocity vector, and $D$ is the distance from the object seen by an OF sensor.

\section{Simulated flying agent}

The behaviour of the "simulated bee" introduced into the in-silico experiments was based on findings which may explain how a flying honeybee controls its speed and avoids walls in tunnel-like environments ([9], [7], [8]). Each translational axis of the bee's dynamic model was uncoupled, as occurs in a quadrotor (Fig. 1A). Each of the bee's translational dynamics can be defined by a first order transfer function with a time constant of $0.22 \mathrm{~s}$ (see [21] for details). Pitch angle, roll angle, and wing stroke amplitude were bounded on the basis of data previously published on bees ([21]). In this indoor study, the simulated agent was not subjected to any wind and could only move in translation.

The present agent was equipped with a set of $10 \mathrm{OF}$ sensors for the doubly tapered tunnel (LMS 1 to LMS 10 in Fig. 1) and with a set of 8 OF sensors (LMS 1 to LMS 8 in Fig. 1) for the tunnel with an abrupt change in width without ceiling: each of these sensors consisted of just two photoreceptors driving a Local Motion Sensor (LMS) based on a "time-of-travel" scheme ([15], [16]). The visual axes of the two adjacent photoreceptors were assumed to be separated by the angle $\Delta \varphi$, and each photoreceptor's angular sensitivity was assumed to be a Gaussoid function with an angular width at half height of $\Delta \rho=\Delta \varphi$. The inter-receptor angles $\Delta \varphi$ were therefore smaller in the frontal or rear parts of the visual system $\left(\Delta \varphi_{45^{\circ}}=\Delta \varphi_{90^{\circ}} \times \sin \left(45^{\circ}\right)=2.82^{\circ}\right)$, than in the lateral, ventral, or dorsal parts $\left(\Delta \varphi_{90^{\circ}}=4^{\circ}\right)$ as observed in the bee's compound eye ([25]). A sine-law gradient can be used either in the horizontal or vertical planes to compute the corresponding inter-receptor angle $\Delta \varphi .6$ OF sensors were located in the horizontal plane oriented at azimuthal angles $\varphi$ of $\pm 45^{\circ}, \pm 90^{\circ}$, and $\pm 135^{\circ}$ (Fig. 1A), and the other 4 were oriented in the vertical plane at elevation angles $\theta$ of $-45^{\circ}, \pm 90^{\circ}$ and $+135^{\circ}$ (Fig. 1B).

\section{VISUAL EVENT-BASED DISCRETE TIME CONTROLLERS}

In this section, we describe how we adapted the eventbased control approach introduced in [17] for visual eventbased OF regulation purposes. As presented in the schematic of the event-based autopilot in Fig. 2, three control input signals were computed when a visual event occurs, corresponding to the three uncoupled translational degrees of freedom on the surge $(x)$, sway $(y)$ and heave axes $(z)$. We consider in this work (i) as a "visual event", an event-related

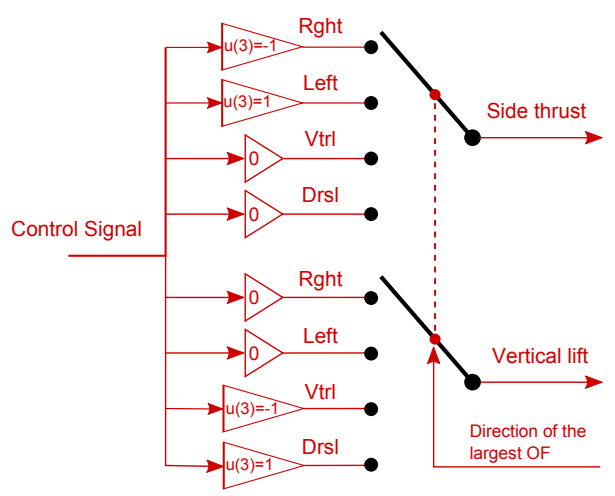

Fig. 3. The control direction selector automatically selects the tunnel surface to be followed (wall, ground or roof) by multiplying the control signal (the output from the Positioning controller) by a direction factor $u(3)$ that depends on the direction of the largest OF signal. Note that the sway and heave dynamics can be driven alternately, depending on which OF (side or vertical) is the largest at any given time. Adapted from [21].

condition based on the visual contrast detection inside the bio-inspired optic flow sensors and (ii) as a "control event", an event-related condition based on optic-flow error signals.

Lead controllers are introduced into the heave and sway control systems to improve their stability. The natural integrator that relates the simulated agent's position to its speed makes both the heave and sway control systems reach a zero steady state error. However, a PI controller is introduced into the surge control system because OF is proportional to speed, but inversely proportional to distance (see (2)). Consequently, we have to add an integral action to cancel the steady state error.

\section{A. Visual event-based PI controller on the surge axis}

A discrete time PI controller was designed. The modelling of the proportional part was quite straightforward, and the backward difference approximation method was used to model the integral part. The resulting code is:

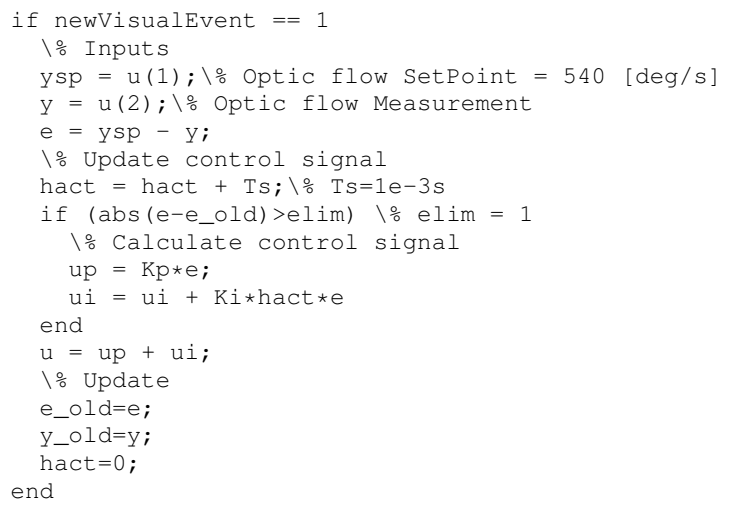

The event-based controller is tuned with the values $K_{p}=$ $20.10^{-3}, K_{i}=6.10^{-2}$ and $T_{s}=1 \mathrm{~ms}$.

B. Visual event-based PD controllers on the sway and the heave axes

Two discrete time PD controllers were also designed to deal with the sway $(y)$ and heave axes $(z)$. Modelling the 
proportional part was quite straightforward, and the backward difference approximation method was used to model the derivative part. The following code was thus obtained for each controller:

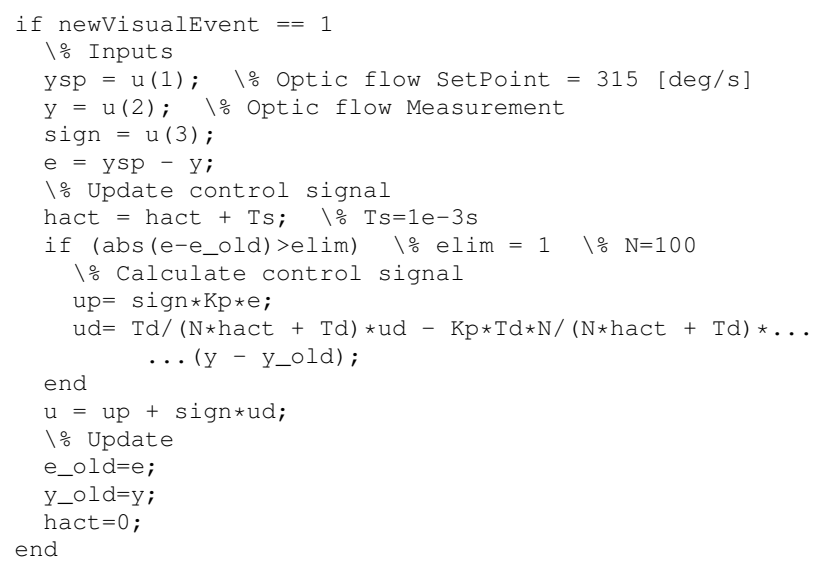

The event-based controllers on the sway axis were tuned with the values $K_{p}=20.10^{-4}, T_{d}=0.1 \mathrm{~s}$, and on the heave axis, they were tuned with the values $K_{p}=20.10^{-3}$, $T_{d}=0.1 \mathrm{~s}$. The input sign was defined on the basis of Fig. 3 adapted from [21]. The parameter elim, was chosen in order to significantly reduce the control updates, while making it possible to perform reference tracking of the OF set-points.

\section{RESULTS}

Frontal and fronto-lateral spatial configurations of the optic flow sensors $\left( \pm 30^{\circ}\right.$ and $\pm 45^{\circ}$, respectively) were tested in the following simulated experiments.

\section{A. Doubly tapered tunnel}

In this simulation, as presented in Fig.2, the following parameters are chosen such as:

$$
\begin{gathered}
k_{1} \omega_{1}=\max \left(\frac{\omega_{L}^{45^{\circ}}}{\sin ^{2}\left(45^{\circ}\right)}, \frac{\omega_{L}^{90^{\circ}}}{\sin ^{2}\left(90^{\circ}\right)}, \frac{\omega_{L}^{135^{\circ}}}{\sin ^{2}\left(135^{\circ}\right)}\right) \\
k_{2} \omega_{2}=\max \left(\frac{\omega_{R}^{-45^{\circ}}}{\sin ^{2}\left(45^{\circ}\right)}, \frac{\omega_{R}^{-90^{\circ}}}{\sin ^{2}\left(90^{\circ}\right)}, \frac{\omega_{R}^{-135^{\circ}}}{\sin ^{2}\left(135^{\circ}\right)}\right) \\
k_{3} \omega_{3}=\max \left(\frac{\omega_{V}^{-45^{\circ}}}{\sin ^{2}\left(45^{\circ}\right)}, \frac{\omega_{V}^{-90^{\circ}}}{\sin ^{2}\left(90^{\circ}\right)}\right) \\
k_{4} \omega_{4}=\max \left(\frac{\omega_{D}^{90^{\circ}}}{\sin ^{2}\left(90^{\circ}\right)}, \frac{\omega_{D}^{135^{\circ}}}{\sin ^{2}\left(135^{\circ}\right)}\right)
\end{gathered}
$$

Each OF output signal $\omega_{i}(i=1,2,3,4)$ is weighted as explained in [13]. The subscripts in $\omega_{L}, \omega_{R}, \omega_{V}$ and $\omega_{D}$ stand for the OF output signal in the left, right, ventral and dorsal directions. In figure 4 , the simulated environment was a doubly tapering tunnel $6 \mathrm{~m}$ long, $1 \mathrm{~m}$ wide, $1 \mathrm{~m}$ high with a slope angle of $7^{\circ}$. Figure $4 \mathrm{~A}$ shows a perspective view of the tunnel. The simulated bee entered the tunnel at zero speed, with the initial coordinates $x_{0}=0.1 \mathrm{~m}$ and various couples/pairs of $y_{0}$ and $z_{0}$ (Fig.4B). Within the first 800 $\mathrm{ms}$, the simulated bee was controlled in the open loop mode
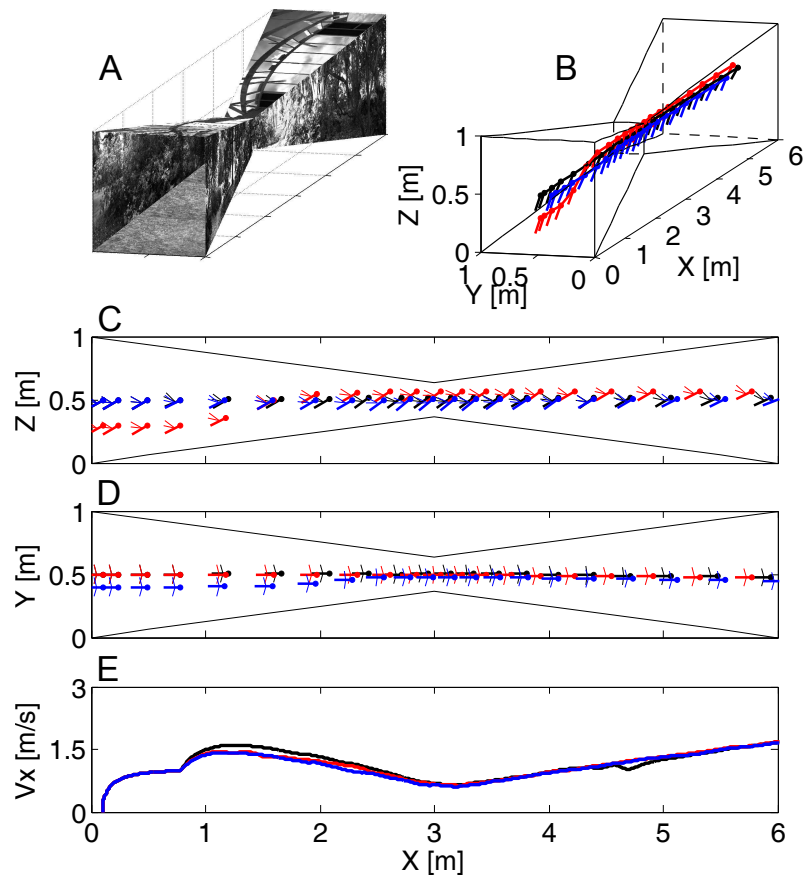

Fig. 4. (A) Perspective view of the doubly tapering tunnel. (B) Simulated bees 3D trajectories starting at the initial coordinates $x_{0}=0.1 \mathrm{~m} ; y_{0}=$ $0.5 \mathrm{~m} ; z_{0}=0.5 \mathrm{~m}$ (in black), $x_{0}=0.1 \mathrm{~m} ; y_{0}=0.4 \mathrm{~m} ; z_{0}=0.5 \mathrm{~m}$ (in blue) and $x_{0}=0.1 \mathrm{~m} ; y_{0}=0.5 \mathrm{~m} ; z_{0}=0.3 \mathrm{~m}$ (in red) plotted every $300 \mathrm{~ms},(\mathrm{C})$ Trajectories in the vertical plane $(x, z)$, and in (D) in the horizontal plane $(x, y)$, plotted every $300 \mathrm{~ms}$. (E) Forward speed $V_{x}$ profiles.

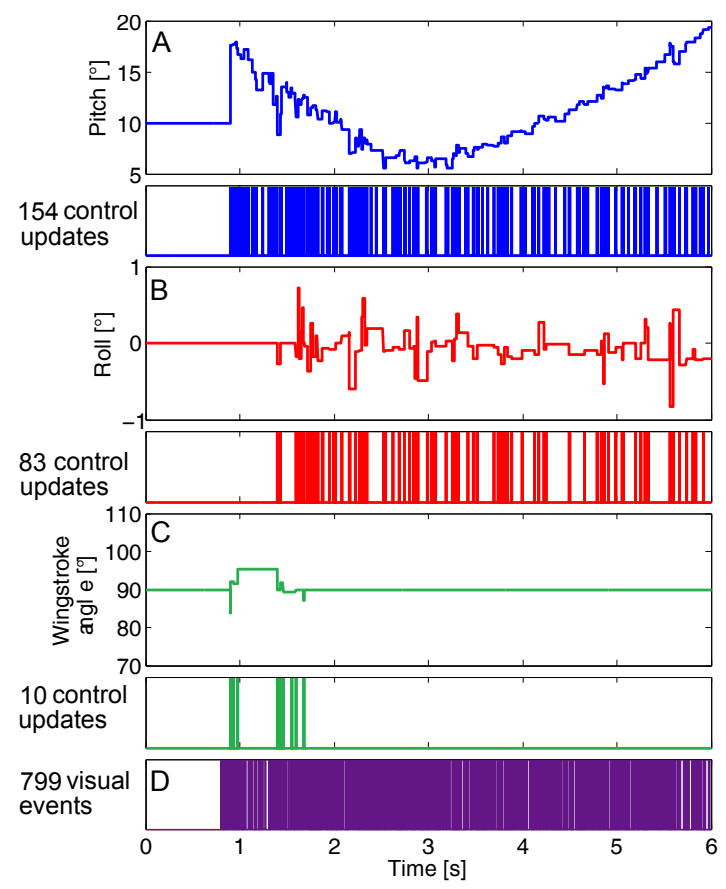

Fig. 5. Simulated control signals, control updates and visual events during a $3 \mathrm{D}$ trajectory starting at the initial coordinates $x_{0}=0.1 \mathrm{~m} ; y_{0}=0.5 \mathrm{~m}$; $z_{0}=0.3 \mathrm{~m}$. (A) The pitch angle is updated 154 times, (B) The roll angle is updated 83 times, (C) The wing stroke angle is updated 10 times, (D) 799 contrast detections stand for the visual events over the simulation.

to enable all the sensors measure the OFs. From the time $800 \mathrm{~ms}$ onwards, the OF was regulated in the closed loop 
mode via the event-based controllers. Figure $4 \mathrm{C}$ shows three trajectories in the vertical plane $(x, z)$ and Fig.4D shows these trajectories in the horizontal plane $(x, y)$, plotted every $300 \mathrm{~ms}$. The simulated bee can be seen to have gradually increased both its ground clearance (Fig.4C) and its right and left clearance (Fig.4D), while the forward speed (Fig.4E) increased automatically up to $1.5 \mathrm{~m} / \mathrm{s}$.

Figure 5 shows the control input signals with a starting point $x_{0}=0.1 \mathrm{~m}, y_{0}=0.5 \mathrm{~m}$ and $z_{0}=0.3 \mathrm{~m}$. The control input signals composed of the pitch, roll and wing stroke angles are also plotted in Fig.5A, 5B and 5C, respectively. During the horizon of simulation of six seconds at the sampling time $T_{s}=1 \mathrm{~ms}$, as shown in Fig.5D, the computation of the condition if (abs (e-e_old)>elim) is activated only 799 times by the visual events. Therefore, it can be seen that the number of control updates is greatly reduced to 154 , 83 and 10, for the pitch, the roll and the wing stroke control signals, respectively. This can be compared with fixed-step PID controllers computed at $1 \mathrm{kHz}$. Figure 6A plots the OF with respect to the set-point of $540^{\circ} / \mathrm{s}$ driving the surge dynamics. In figure $6 \mathrm{~B}$, the $\mathrm{OF}$ is plotted with respect the set-point of $315^{\circ} / \mathrm{s}$ driving the sway and heave dynamics. Both systems of OF regulation gave satisfactory results.
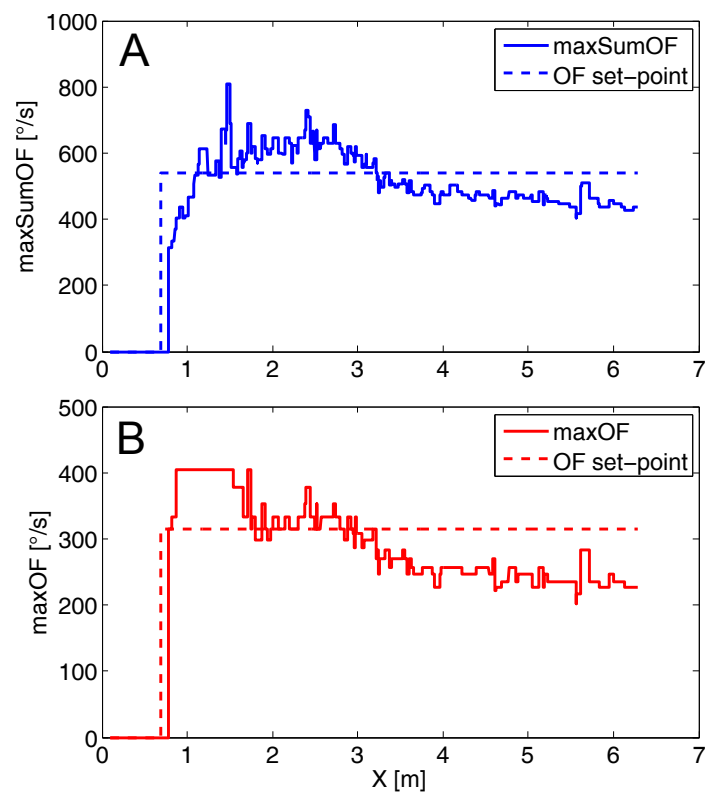

Fig. 6. Simulated systems of OF regulation in the case of a 3D trajectory starting at the initial coordinates $x_{0}=0.1 \mathrm{~m} ; y_{0}=0.5 \mathrm{~m} ; z_{0}=0.3 \mathrm{~m}$. (A) Forward feedback signal determined by taking the largest sum of two diametrically opposed OF sensors (horizontal OF sensors, vertical OF sensors). (B) Positioning feedback signal determined by taking the largest output from the OF sensors (right OF sensors; left OF sensors, ventral OF sensors, dorsal OF sensors).

\section{B. Tunnel with an abrupt change in width}

In figure $7 \mathrm{~A}$, the simulated environment was a tunnel with an abrupt change in width whose dimensions are $12 \mathrm{~m}$ long, $1 \mathrm{~m}$ wide and $1 \mathrm{~m}$ high. The abrupt change is located at midway. Within the first $1.5 \mathrm{~s}$, the simulated bee was controlled in the open loop mode to enable all the sensors

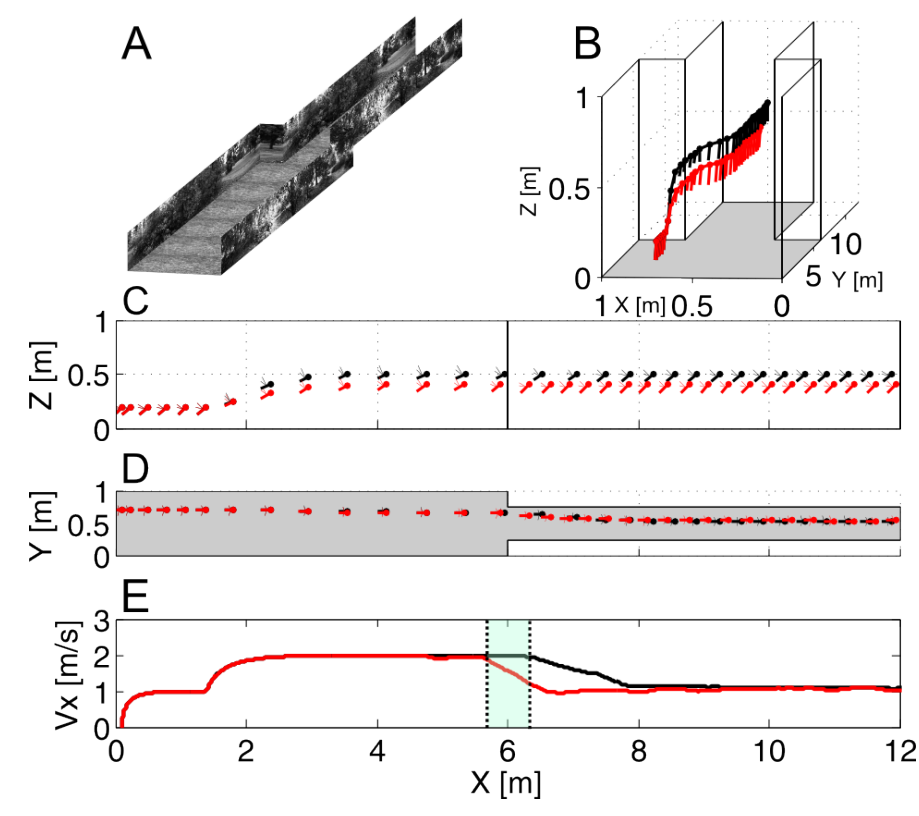

Fig. 7. Comparison between two spatial configurations of optic flow sensors: frontal configuration $\left( \pm 30^{\circ}\right.$, in red) and fronto-lateral configuration ( $\pm 45^{\circ}$, in black) (A) Perspective view of the tunnel with an abrupt change in width. (B) Two simulated bees 3D trajectories starting at the same initial coordinates $x_{0}=0.1 \mathrm{~m} ; y_{0}=0.7 \mathrm{~m} ; z_{0}=0.2 \mathrm{~m}$ plotted every $300 \mathrm{~ms}$. In black, 6 OF sensors were located in the horizontal plane oriented at azimuthal angles of $\pm 45^{\circ}, \pm 90^{\circ}$, and $\pm 135^{\circ}$ (Fig. 1B), and the other 4 were oriented in the vertical plane at elevation angles of $-45^{\circ}, \pm 90^{\circ}$ and $+135^{\circ}$ (Fig. 1C). In red, the sensors are located in the horizontal plane oriented at azimuthal angles of $\pm 30^{\circ}, \pm 90^{\circ}$, and $\pm 150^{\circ}$, and in the vertical plane at elevation angles of $-30^{\circ}, \pm 90^{\circ}$ and $+150^{\circ}$. (C) Trajectories in the vertical plane $(x, z)$, and in (D) in the horizontal plane $(x, y)$. (E) Forward speed $V_{x}$ profiles.

measure the OF. From the time $1.5 \mathrm{~s}$ onwards, the OF was regulated in the closed loop mode combining both visual and control events. Figure 7B shows two simulated bees 3D trajectories starting at the same initial coordinates $x_{0}=$ $0.1 \mathrm{~m} ; y_{0}=0.7 \mathrm{~m} ; z_{0}=0.2 \mathrm{~m}$ plotted every $300 \mathrm{~ms}$ for two different sets of parameters, in black using (3)-(5), in red using (7)-(9).

$$
\begin{gathered}
k_{1} \omega_{1}=\max \left(\frac{\omega_{L}^{30^{\circ}}}{\sin ^{2}\left(30^{\circ}\right)}, \frac{\omega_{L}^{90^{\circ}}}{\sin ^{2}\left(90^{\circ}\right)}, \frac{\omega_{L}^{150^{\circ}}}{\sin ^{2}\left(150^{\circ}\right)}\right) \\
k_{2} \omega_{2}=\max \left(\frac{\omega_{R}^{-30^{\circ}}}{\sin ^{2}\left(30^{\circ}\right)}, \frac{\omega_{R}^{-90^{\circ}}}{\sin ^{2}\left(90^{\circ}\right)}, \frac{\omega_{R}^{-150^{\circ}}}{\sin ^{2}\left(150^{\circ}\right)}\right) \\
k_{3} \omega_{3}=\max \left(\frac{\omega_{V}^{-30^{\circ}}}{\sin ^{2}\left(30^{\circ}\right)}, \frac{\omega_{V}^{-90^{\circ}}}{\sin ^{2}\left(90^{\circ}\right)}\right)
\end{gathered}
$$

$k_{4} \omega_{4}=0$ since the dorsal OF is equal to zero (the tunnel has no ceiling). While figures $7 \mathrm{C}$ and 7D exhibit similar results in the vertical and horizontal planes, Fig.7E shows a decrease in the forward speed $V_{x}$ before the abrupt change with azimuthal angles equal to $\pm 30^{\circ}$. The constriction point is anticipated sooner than for azimuthal angles equal to $\pm 45^{\circ}$. Moreover one can note that the forward control loop is much more faster than the position control loop as depicted in Figs.7D and 7E. Consequently, in the future simulations, 


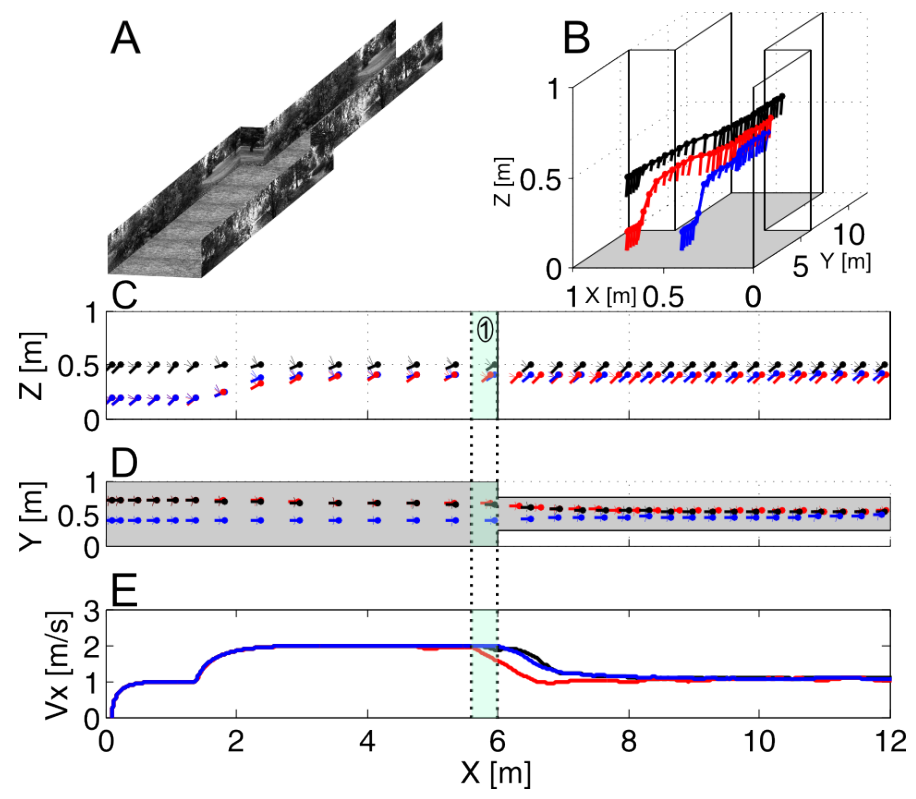

Fig. 8. Comparison between three sets of initial conditions where the six OF sensors were located in the horizontal plane oriented at azimuthal angles of $\pm 30^{\circ}, \pm 90^{\circ}$, and $\pm 150^{\circ}$, and the other 4 were oriented in the vertical plane at elevation angles of $-30^{\circ}, \pm 90^{\circ}$ and $+150^{\circ}$. (A) Perspective view of the tunnel with an abrupt change in width. (B) Simulated bees $3 \mathrm{D}$ trajectories starting at the initial coordinates $x_{0}=0.1 \mathrm{~m} ; y_{0}=0.7 \mathrm{~m}$; $z_{0}=0.5 \mathrm{~m}$ (in black), $x_{0}=0.1 \mathrm{~m} ; y_{0}=0.4 \mathrm{~m} ; z_{0}=0.5 \mathrm{~m}$ (in blue) and $x_{0}=0.1 \mathrm{~m} ; y_{0}=0.7 \mathrm{~m} ; z_{0}=0.2 \mathrm{~m}$ (in red) plotted every $300 \mathrm{~ms}$. (C) Trajectories in the vertical plane $(x, z)$, and in (D) in the horizontal plane $(x, y)$, plotted every $300 \mathrm{~ms}$. (E) Forward speed $V_{x}$ profiles.

the parameters given by (7)-(9) and $k_{4} \omega_{4}=0$ are chosen. Figure $8 \mathrm{C}$ shows 3 trajectories in the vertical plane $(x, z)$ and Fig.8D shows these trajectories in the horizontal plane $(x, y)$, plotted every $300 \mathrm{~ms}$. The simulated bee can be seen to have gradually increased the forward speed (Fig.8E) up to $2 \mathrm{~m} / \mathrm{s}$ and decreased the forward speed as soon as the abrupt change was detected thanks to the OF sensors oriented at the azimuthal angles $\varphi$ of $\pm 30^{\circ}$. Figure 9 shows the control input signals, the control updates and the visual events with $x_{0}=0.1 \mathrm{~m}, y_{0}=0.7 \mathrm{~m}$ and $z_{0}=0.2 \mathrm{~m}$. One can note that the computation of the condition on the error leading the eventbased controllers is activated 1030 times by the visual events (see Fig.9D). It results low numbers of control updates, 236 for the pitch, 130 for the roll and 25 for the wing stroke angles. Figure 10A plots the OF with respect to the setpoint of $540^{\circ} / \mathrm{s}$ driving the surge dynamics. In figure $10 \mathrm{~B}$, the $\mathrm{OF}$ is plotted with respect the set-point of $315^{\circ} / \mathrm{s}$ driving the sway and heave dynamics. The event-based controllers cancel the disturbance introduced by the abrupt change in tunnel width. Using OF measurement coming from $30^{\circ}$ to $150^{\circ}$ to control the speed of the "simulated bee" allows it to reduce its speed before the constriction point (blue shaded zone in Fig. 8E), as bees did in similar tunnel configuration ([9], [10]).

\section{CONCLUSIONS}

In this paper, we present an innovative visual event-based autopilot mimicking the direct Optic Flow (OF) feedback

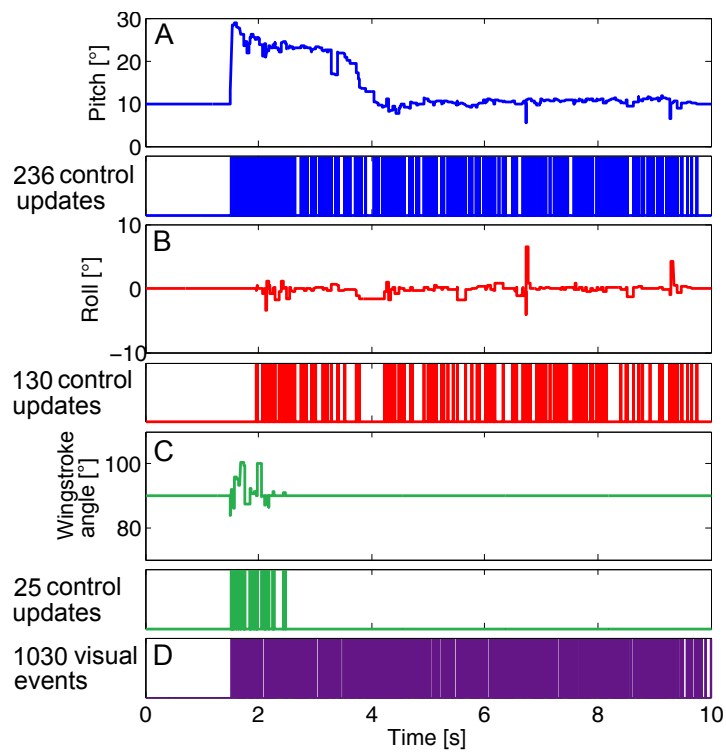

Fig. 9. Simulated control signals, control updates and visual events during a 3D trajectory starting at the initial coordinates $x_{0}=0.1 \mathrm{~m} ; y_{0}=0.7 \mathrm{~m}$; $z_{0}=0.2 \mathrm{~m}$. (A) The pitch angle is updated 236 times, (B) The roll angle is updated 130 times, (C) The wing stroke angle is updated 25 times, (D) 1030 constrast detections stand for the visual events over the simulation in the tunnel with an abrupt change in width.
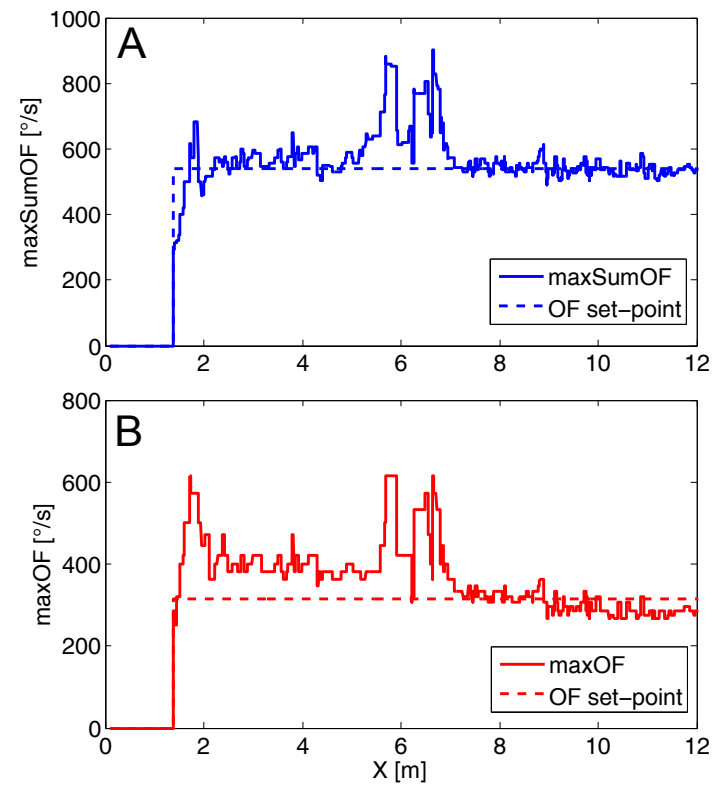

Fig. 10. Simulated systems of OF regulation in the case of a 3D trajectory starting at the initial coordinates $x_{0}=0.1 \mathrm{~m} ; y_{0}=0.7 \mathrm{~m} ; z_{0}=0.2 \mathrm{~m}$

control loops which may occur in honeybees. Likewise, honeybees tiny brains contain smart OF sensors, called Velocity-Tuned motion-sensitive descending neurons (or VT neurons) which are spiking neurons sensitive to the OF ([26]): each spike can be taken to constitute a visual event occurring in the insects neuronal system. Our proposed visual event-based control system combines both error signals and visual events. In a near future, it will be likely to become a major tool which can be used to understand more clearly how insects process the event-based visual information they 
receive in order to react appropriately: this information ranges from optical contrasts and local motion detection to self-induced wing movements generating the insects $3 \mathrm{D}$ movements in any tunnel configuration. This is the first time we tended to explain using a simulation that a decrease in the forward speed before the abrupt change in tunnel width can be observed only by considering OF measurements coming from frontal azimuthal angles $\pm 30^{\circ}$ instead of fronto-lateral azimuthal angles $\pm 45^{\circ}$ (Fig.7E). These results are particularly consistent with bumblebees behavior in similar situations ([9], [10]). However, no difference in the simulation is observed in the lateral displacement with both spatial configurations (Fig.7D) before reaching the constriction point, which is also similarly observed in bumblebees ([10]). The bumblebees behavior across an abrupt change in tunnel width may be therefore explained jointly by their dynamic capabilities and their viewing angles.

The results of the present in-silico experiments show that the visual event-based control system based on OF consisting in updating the control signals solely when visual contrasts are detected provides a suitable basis for traveling along unknown tunnels endowed with cross-section changes. It is now proposed to perform further studies involving a more sophisticated OF-based autopilot traveling along more complex tunnels, rooms, or mazes, as well as to implement the visual event-based ALIS autopilot in the near future onboard a 25-gram nano-quadrotor ([3]).

\section{ACKNOWLEDGMENT}

We thank R. Brinkworth and D. O'Carroll (Adelaide Uni.) for kindly making their panoramic images available to us.

\section{REFERENCES}

[1] A. Kushleyev, D. Mellinger, C. Powers, and V. Kumar, "Towards a swarm of agile micro quadrotors," Autonomous Robots, vol. 35, no. 4, pp. 287-300, 2013.

[2] K. Y. Ma, P. Chirarattananon, S. B. Fuller, and R. J. Wood, "Controlled flight of a biologically inspired, insect-scale robot," Science, vol. 340, no. 6132, pp. 603-607, 2013.

[3] O. Dunkley, J. Engel, J. Sturm, and D. Cremers, "Visual-inertial navigation for a camera-equipped 25g nano-quadrotor," in IROS2014 aerial open source robotics workshop, 2014.

[4] D. Floreano and R. J. Wood, "Science, technology and the future of small autonomous drones," Nature, vol. 521, no. 7553, pp. 460-466, 2015.

[5] J. Gibson, The perception of the visual world. Boston: Houghton Mifflin, 1950.

[6] J. J. Koenderink and A. J. van Doorn, "Facts on optic flow," Biological cybernetics, vol. 56, no. 4, pp. 247-254, 1987.

[7] G. Portelli, F. Ruffier, F. L. Roubieu, and N. Franceschini, "Honeybees' speed depends on dorsal as well as lateral, ventral and frontal optic flows," PLoS One, vol. 6, no. 5, p. e19486, 2011.

[8] M. V. Srinivasan, "Visual control of navigation in insects and its relevance for robotics," Current opinion in neurobiology, vol. 21, no. 4, pp. 535-543, 2011.

[9] E. Baird, T. Kornfeldt, and M. Dacke, "Minimum viewing angle for visually guided ground speed control in bumblebees," Journal of Experimental Biology, vol. 213, no. 10, pp. 1625-1632, 2010.

[10] N. Linander, M. Dacke, and E. Baird, "Bumblebees measure optic flow for position and speed control flexibly within the frontal visual field," Journal of Experimental Biology, vol. 218, no. 7, pp. 10511059, 2015.

[11] A. Beyeler, J.-C. Zufferey, and D. Floreano, "Vision-based control of near-obstacle flight," Autonomous robots, vol. 27, no. 3, pp. 201-219, 2009.
[12] P. A. Shoemaker, A. M. Hyslop, and J. S. Humbert, "Optic flow estimation on trajectories generated by bio-inspired closed-loop flight," Biological cybernetics, vol. 104, no. 4-5, pp. 339-350, 2011.

[13] F. L. Roubieu, J. R. Serres, F. Colonnier, N. Franceschini, S. Viollet, and F. Ruffier, "A biomimetic vision-based hovercraft accounts for bees complex behaviour in various corridors," Bioinspiration \& biomimetics, vol. 9, no. 3, p. 036003, 2014.

[14] F. Expert and F. Ruffier, "Flying over uneven moving terrain based on optic-flow cues without any need for reference frames or accelerometers," Bioinspiration \& biomimetics, vol. 10, no. 2, p. 026003, 2015.

[15] N. Franceschini, A. Riehle, and A. Le Nestour, "Directionally selective motion detection by insect neurons," in Facets of vision. Springer, 1989, pp. 360-390.

[16] E. Vanhoutte, S. Mafrica, F. Ruffier, R. J. Bootsma, and J. Serres, "Time-of-travel methods for measuring optical flow on board a micro flying robot," Sensors, vol. 17, no. 3, p. 571, 2017.

[17] S. Durand and N. Marchand, "Further results on event-based PID controller," in Control Conference (ECC), 2009 European, Aug 2009, pp. 1979-1984.

[18] X. Clady, C. Clercq, S.-H. Ieng, F. Houseini, M. Randazzo, L. Natale, C. Bartolozzi, and R. Benosman, "Asynchronous visual event-based time-to-contact," Neuromorphic Engineering Systems and Applications, p. 51, 2015.

[19] M. B. Milde, O. J. N. Bertrand, R. Benosman, M. Egelhaaf, and E. Chicca, "Bioinspired event-driven collision avoidance algorithm based on optic flow," in Event-based Control, Communication, and Signal Processing (EBCCSP), 2015 International Conference on. IEEE, 2015, pp. 1-7.

[20] A. Censi and D. Scaramuzza, "Low-latency event-based visual odometry," in Robotics and Automation (ICRA), 2014 IEEE International Conference on. IEEE, 2014, pp. 703-710.

[21] G. Portelli, J. Serres, F. Ruffier, and N. Franceschini, "Modelling honeybee visual guidance in a 3-D environment," Journal of PhysiologyParis, vol. 104, no. 1, pp. 27-39, 2010.

[22] J. Serres, T. Raharijaona, E. Vanhoutte, and F. Ruffier, "Event-based visual guidance inspired by honeybees in a $3 \mathrm{~d}$ tapered tunnel," in Second International Conference on Event-Based Control, Communication and Signal Processing EBCCSP 2016, 2016.

[23] R. S. Brinkworth and D. C. Carroll, "Biomimetic motion detection," in Intelligent Sensors, Sensor Networks and Information, 2007. ISSNIP 2007. 3rd International Conference on. IEEE, 2007, pp. 137-142.

[24] S. Viollet and J. Zeil, "Feed-forward and visual feedback control of head roll orientation in wasps (polistes humilis, vespidae, hymenoptera)," The Journal of experimental biology, vol. 216, no. 7, pp. 1280-1291, 2013.

[25] R. Seidl, Die Sehfelder und Ommatidien-Divergenzwinkel von Arbeiterin, Königin und Drohn der Honigbiene (Apis mellifica). $\mathrm{PhD}$ thesis, Technische Hochschule Darmstadt, Germany, 1982.

[26] M. R. Ibbotson, "Evidence for velocity-tuned motion-sensitive descending neurons in the honeybee." Proc $R$ Soc of London B, vol. 268, no. 1482, pp. 2195-2201, Nov 2001. 